\title{
Ruptured Hydatid Cyst of the Liver with Biliary Obstruction - Endoultrasound Findings
}

\author{
Shiran Shetty, Mohan Krishnan, Krishnaveni Janathan, Venkatakrishnan Leelakrishnan \\ Department of Gastroenterology, PSG Institute of Medical Science and Research, Peelamedu, Coimbatore, Tamil Nadu, India
}

A 50-year-old male patient was admitted with abdominal pain and jaundice of 2 weeks duration. The patient had no previous significant medical illness. Physical examination displayed icterus with palpable liver. Laboratory findings revealed eosinophilia; serum bilirubin, $5.5 \mathrm{mg} / \mathrm{dl}$; serum alkaline phosphatase, 265 IU/l (normal 80-160 IU/1); SGOT, $77 \mathrm{IU} / 1$; and SGPT, $112 \mathrm{IU} / \mathrm{l}$.

Abdominal ultrasongraphy (USG) revealed moderate hepatomegaly with dilated common bile duct (CBD), cyst in the liver with echogenic walls and echogenic irregular areas in the interior (Figure 1). Endoscopic ultrasound (EUS) showed multiple floating hyperechoic structures of varying sizes and shapes in the CBD (Figure 2) and multiple cysts in the liver. The structures were rounded, oval and leaf-like in shape (Figure 2). These findings were suggestive of ruptured hydatid cyst with biliary obstruction. Endoscopic retrograde

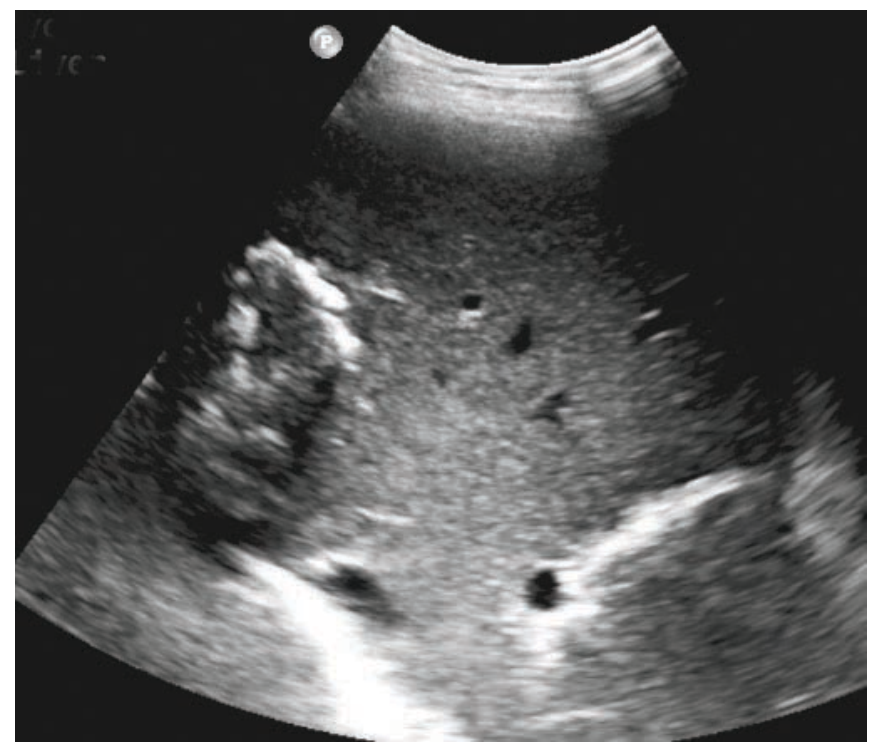

Figure 1: Abdominal ultrasound showing a large hydatid cyst

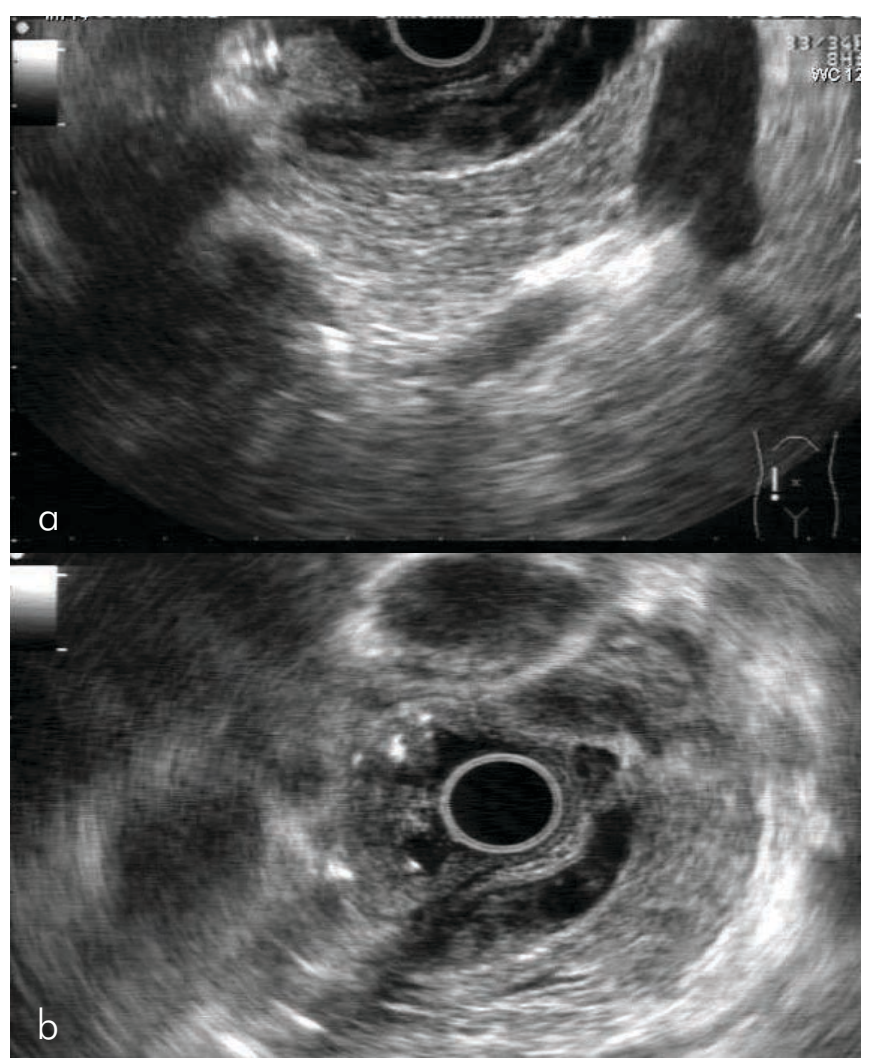

Figure 2: (a) Endoultrasound showing multiple floating hyperechoic structures of varying sizes and shapes in the common bile duct (b) Notice these structures has changed their sizes and shapes.

Reprints requests and correspondence:

Dr Shiran Shetty, MD, DM.

Assistant Professor

Department of Gastroenterology

PSG Institute of Medical Science and Research

Peelamedu, Coimbatore -641004, Tamil Nadu - INDIA

Email:drshiran@gmail.com

Mobile: +919790306917 

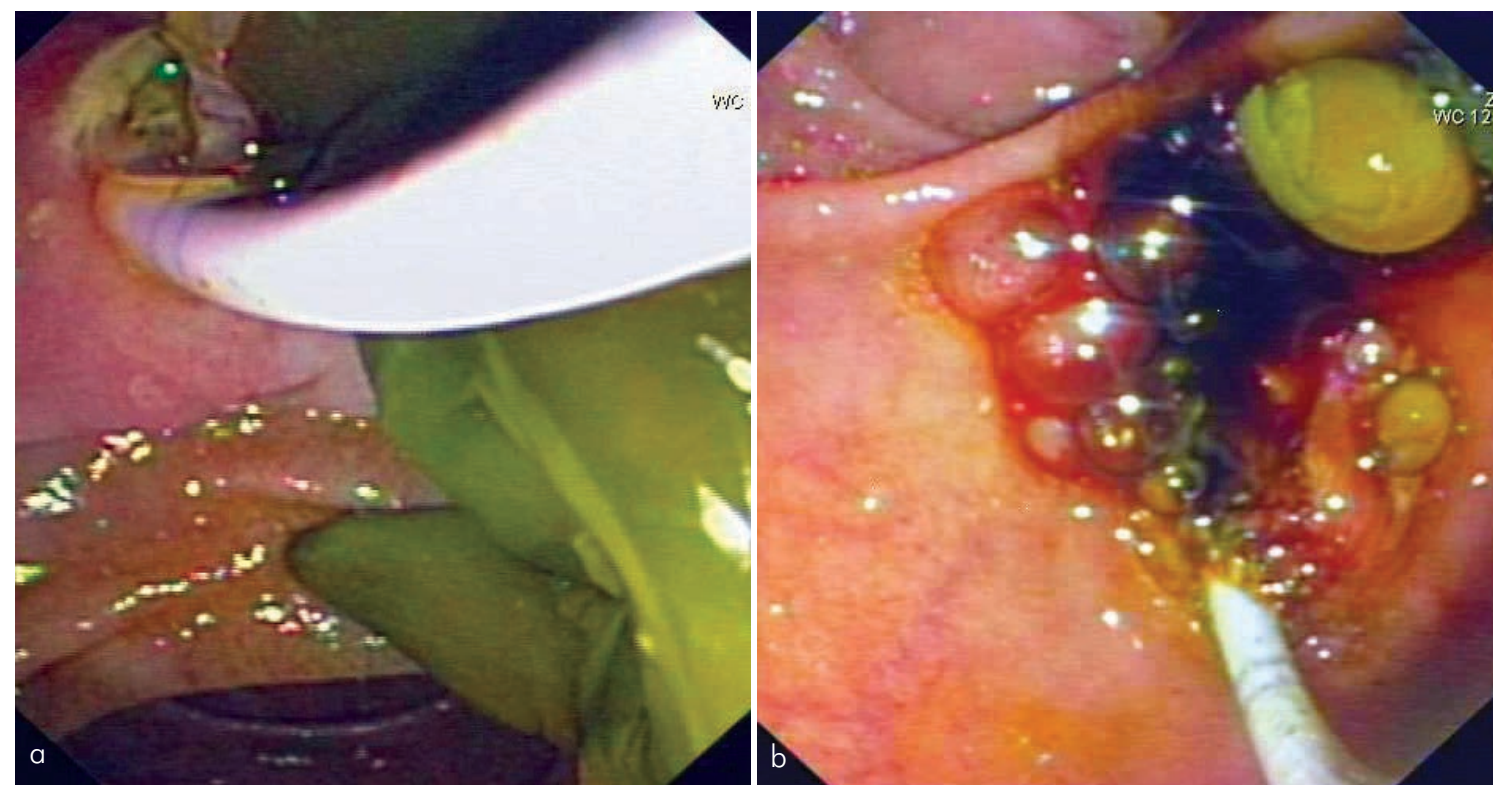

Figure 3a \& 3b: ERCP revealing multiple hydatid brood capsules, membranes and hydatid debris

cholangiopancreatography (ERCP) revealed multiple filling defects as seen in endoultrasound. After an sphincterotomy, hydatid brood capsules and membranes were removed and CBD stented (Figure 3). Patient was further treated with albendazole at a dose of $10 \mathrm{mg} / \mathrm{kg} /$ day (400mg twice a day) and surgery was done after 3 weeks.

Hydatid disease continues to be a health problem in sheep-raising areas of the world. It is caused by a parasite, Echinococcus granulosus. It involves the liver in about $70 \%$ of cases. Intrabiliary rupture, transthoracic extension and peritoneal dissemination are well-known complications of liver hydatid cyst. Intrabiliary rupture is a serious complication and reported to occur in $4-25 \%$ of cases. It presents with obstructive jaundice, cholangitis, pancreatitis and septicemia. Accurate preoperative diagnosis is essential to reduce morbidity and mortality. ${ }^{1,2}$ Ultrasonography, ${ }^{3,4}$ endoscopic retrograde cholangiopancreatography, ${ }^{4}$ computer tomography ${ }^{5}$ and magnetic resonance imaging and cholangiopancreatography ${ }^{6}$ are well-established imaging modalities that have been used for the diagnosis of intrabiliary rupture of liver hydatid cyst. Endoultrasound features describes in our case are not different from those reported at endoscopic retrograde cholangiopancreatography or magnetic resonance cholangiopancreatography.

\section{References}

1. Atli M, Kama NA, Yuksek YN . Intrabiliary rupture of a hepatic hydatid cyst associated clinical factors and management. Arch Surg 2001;136:1249-55.

2. Bedirli A, Sakrak O, Sozuer EM, Kerek M, Ince O. Surgical management of spontaneous Intrabiliary rupture of hydatid liver cysts. Surg Today 2002;32:594-7.

3. Camunez Camunez F, Simo G, Robledo R, et al. Ultrasound diagnosis of ruptured hydatid cyst of the liver with biliary obstruction. Gastrointest Radiol 1986;11:330-3.

4. Zargar SA, Khuroo MS, Khan BA, Dar MY, Alai MS, Koul P. Intrabiliary rupture of hepatic hydatid cyst: sonographic and cholangiographic appearances. Gastrointest Radiol 1992;17:41-5

5. Tsitouridis J, Kouklakis G, Tsitouridis K, Melidis D, Krokos N, Emmanoyilidoy M. Intrabiliary obstruction due to ruptured hepatic hydatid cyst: evaluation with computed tomography and magnetic resonance imaging. Dig Endosc 2001; 13:7.

6. Ormeci N, Fitoz S, Erden I, Tanju S, Gency Y. Intrabiliary rupture of hepatic hydatid cysts: diagnostic accuracy of MR cholangiopancreatography. AJR 2007;189:84-9.

Source of support: Nil; Conflict of interest: none declared 